\title{
TMS-Induced Seizure during FDA- Approved Bilateral DMPFC Protocol for Treating OCD: A Case Report
}

\author{
Georgios Mikellides ${ }^{a}$ b $\quad$ Panayiota Michael ${ }^{b}$ Teresa Schuhmann ${ }^{a}$ \\ Alexander T Sack ${ }^{a}$ c \\ aFaculty of Psychology and Neuroscience, Maastricht University, Maastricht, The \\ Netherlands; ${ }^{b}$ Cyprus rTMS Centre, Larnaca, Cyprus; ${ }^{\mathrm{c} D e p a r t m e n t ~ o f ~ P s y c h i a t r y ~ a n d ~}$ \\ Neuropsychology, School for Mental Health and Neuroscience (MHeNs), Brain+Nerve \\ Centre, Maastricht University Medical Centre+ (MUMC+), Maastricht, The Netherlands
}

\section{Keywords}

Obsessive-compulsive disorder - Repetitive transcranial magnetic stimulation .

High-frequency repetitive transcranial magnetic stimulation - Deep brain stimulation .

Dorsomedial prefrontal cortex · Seizure induction · Transcranial magnetic

stimulation-induced seizure

\section{Abstract}

Repetitive transcranial magnetic stimulation (rTMS) is a noninvasive brain stimulation therapy that has become a method of choice for the treatment of several neuropsychiatric disorders such as depression and OCD. It is considered to be a safe and well-tolerated treatment, with only few side effects. The most serious adverse event during any rTMS treatment is the potential induction of a seizure. rTMS has shown very encouraging results for treatment-resistant $O C D$, although the optimal target area and the stimulation frequency are still matters of controversy. Here, we present a 19-year-old female patient with OCD who experienced seizure during the 7th session of her rTMS treatment using the FDA-approved $20-\mathrm{Hz}$ protocol for OCD applied bilaterally over the left and right DMPFC using a double-cone coil. Nonetheless, it still unknown whether the seizure occurred as a consequence of rTMS, as the patient was also in a specific seizure risk group. Future reviews are needed to further clarify the mechanisms that may trigger seizures during rTMS treatments in order to reduce the likelihood of rTMS-induced seizures. 


\section{Introduction}

Repetitive transcranial magnetic stimulation (rTMS) is a noninvasive brain stimulation method that has shown to be clinically effective in treating various neuropsychiatric disorders, in particular depression [1] and obsessive-compulsive disorder (OCD) [2]. rTMS delivers electromagnetic pulses to selective areas of the cerebral cortex using an insulated electromagnetic coil [3]. Depending on the stimulation frequency, rTMS may increase or decrease cortical excitability [3]. The clinical efficacy of TMS in treating depression is by now undisputed, and the FDA-approved depression protocol targeting the dorsolateral prefrontal cortex (DLPFC) is widely recognized and established, with costs for TMS depression treatment being reimbursed by health insurances in a growing number of countries. In a similar vein, also for the treatment of OCD, rTMS has shown encouraging results [2,4]. However, unlike in the case of depression, the optimal target region in the brain for treating OCD with rTMS remains a question of uncertainty. A series of recent studies focused on the effects of stimulating the supplementary motor area (SMA) [5], orbitofrontal cortex (OFC) [6], and DLPFC [7] in treating OCD symptoms. In 2018, the US Food and Drug Administration (FDA) approved the Brainsway Deep Transcranial Magnetic Stimulation System for treatment of OCD [8]. More recently, in 2020, the FDA cleared MagVenture TMS Therapy for adjunct treatment of OCD. This clearance was based on a prospective multicenter randomized double-blind placebo-controlled trial [9]. In this FDA-approved OCD protocol, TMS is applied bilaterally over the left and right dorsomedial prefrontal cortex (DMPFC) using a double-cone coil (specifically designed to achieve deeper stimulation penetration) and applying a high-frequency $20-\mathrm{Hz}$ rTMS sequence. This is different from the other OCD protocols described in the literature, where standard TMS coils are used to stimulate either SMA [5] or DLPFC [7] with a low-frequency rTMS protocol.

Despite its clinical efficacy, also the tolerability of rTMS is of utmost importance in evaluating its clinical usability. Fortunately, rTMS has been shown to be very well tolerated with very few to no side effects in most patients. The most serious adverse event during any rTMS treatment is the potential induction of a seizure. However, the occurrence of seizures cases is extremely low ( $<1$ in 60,000 sessions) in patients without specific risk factors such as congenital epilepsies or anatomical/brain damages [10]. A recent review of the literature on this topic highlights that the presence of neuropsychiatric diseases associated with structural cerebral damage (e.g., traumatic brain injury and stroke), general factors (e.g., stress, sleep deprivation, and increased alcohol consumption), and some medical conditions (e.g., metabolic abnormalities and alcohol withdrawal) may increase the risk for seizures [11]. Until early 2020, a total number of 41 seizures were reported: 13 were healthy individuals and 28 were patients with clinical conditions (like psychiatric disorders and stroke). Regarding the stimulation frequency, 51\% of these seizures occurred during HF-rTMS [12].

In addition to the frequency, also the location or deeper penetrating nature of some OCD protocols may affect the likelihood of seizure induction by TMS. OCD, in this sense, represents a good opportunity to document the occurrence of seizures across the various available and used treatment protocols (across regions from pre-SMA, DLPFC, and orbitofrontal cortex to bilateral DMPFC), protocols (from 1 to $20 \mathrm{~Hz}$ ), and coils (from standard figure-8 coils to double-cone coils to $\mathrm{H}$ coils). This documentation will be informative in not only assessing the clinical efficacy of these various OCD rTMS protocols but also with regard to their tolerability and risk profile. This explicitly should include single case reports from naturalistic clinical settings. In this spirit, we here present the case of a 19-year-old female patient with OCD who experienced seizure during the 7th session of her rTMS treatment using the FDA-approved bilateral DMPFC 20-Hz protocol applied with a double-cone TMS coil.

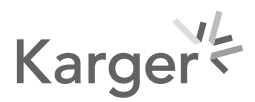



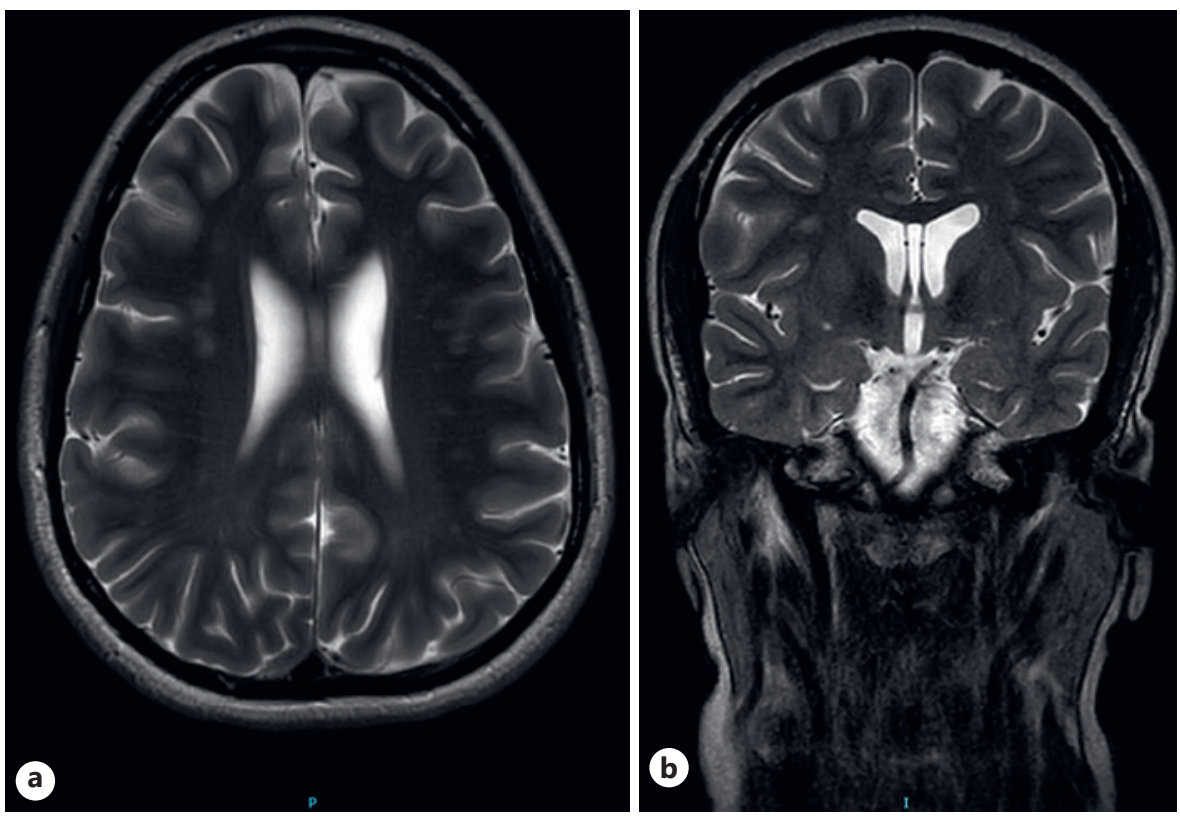

Fig. 1. Brain MRI scans 2 weeks after the first seizure episode. The exam showed several focal lesions of a few millimeters in diameter in the periventricular and subcortical white matter of the frontal and parietal lobes and in the corpus callosum.

\section{Case Report/Case Presentation}

A 19-year-old female patient presented with OCD. This patient has also been diagnosed with depression, Pierre Robin sequence, and mild mental retardation. First, the patient received rTMS for her depressive and anxiety symptoms. Prior to the TMS therapy, the treating psychiatrist followed a pre-rTMS treatment evaluation of the patient, indicating no seizure risk factors. Written informed consent for the rTMS treatment was obtained from the patient. She underwent rTMS treatment targeting the bilateral DLPFC, with the TMS coil being placed over the left and right DLPFC for iTBS and cTBS protocols, respectively. These theta burst protocols were applied using a figure-8 coil and consisted of the following parameters: iTBS: $5 \mathrm{~Hz}, 20$ trains with 8-s intertrain interval, 10 pulses per train, 200 pulses per session, and total duration 3:08 min; cTBS: 20 trains with 0.2-s intertrain interval, 10 pulses per train, 200 pulses per session, and total duration $40 \mathrm{~s}$. The stimulation intensity was set at $120 \%$ of the resting motor threshold. The patient received a total of 30 rTMS sessions. In addition to the rTMS treatment, she was taking at intervals some of the following medications: escitalopram, risperidone, lorazepam, promethazine, and olanzapine. After the completion of the treatment period, the mother of the patient reported the following changes: better attitude toward her family members, more kindness, gentle reactions, rule-following, improvement in self-care, restoration of empathy, and better appetite. The patient continued with approximately 20 maintenance rTMS sessions once a week or once a month depending on her availability.

For a period of 18 months, the patient was free of medications, but then she started having more severe OCD symptoms. Following this, the patient underwent rTMS treatment for OCD using the FDA-approved protocol for OCD [9] with a double-cone coil (Cool D-B80 coil). The rTMS protocol was administered at $20 \mathrm{~Hz}$ and contained 50 trains (40 pulses per train) and 20-s intertrain interval. A total of 2,000 pulses were given per session for 17:58 min. The double-cone coil was placed over the bilateral DMPFC situated $4 \mathrm{~cm}$ in front of the leg motor threshold spot, and the stimulation intensity was set at $100 \%$ of the leg motor 
threshold. The patient was on treatment with clonazepam ( $0.5 \mathrm{mg} /$ day), quetiapine $(600 \mathrm{mg} /$ day), and sertraline (100 mg/day) during the new rTMS treatment for OCD. No adverse events were reported during the first 6 sessions.

During the 7th session, the patient experienced a seizure lasting approximately 2-3 min which was accompanied by a fast and tonic fall, facial grimacing, lateral eye deviation, tonguebiting, body shaking, and generalized tonic-clonic movements. The TMS technician stopped the treatment immediately and informed the treating psychiatrist. Until the seizure ended, the TMS technician and the psychiatrist were on the patient's side and put the patient in a lateral decubitus position. Subsequently, the psychiatrist performed a normal neurological examination and checked the patient's breathing and heart rate. An increased heart rate (120 beats per min) was presented immediately after the seizure ended. Her respiration and heart ( 90 beats per $\mathrm{min}$ ) rate returned to normal levels after approximately $10 \mathrm{~min}$, and no other adverse events like sweating or vomiting were presented. Her postictal symptoms were confusion and headache. The patient was able to respond to verbal instructions and was discharged home. No risk factors for TMS-provoked seizures were observed on that day $[10,11]$.

An MRI measurement was performed 18 months prior to the seizure episode and 2 weeks after the seizure episode (shown in Fig. 1). No visual differences were presented between them. Specifically, both of these MRIs showed several focal lesions of a few millimeters in diameter in the periventricular and subcortical white matter of the frontal and parietal lobes and in the corpus callosum. The patient continued the rTMS treatments 8 days after the seizure induction. The intensity of the treatment was reduced to $70 \%$ of the leg motor threshold. No adverse events were presented during the first 5 days with the new reduced intensity. The patient continued with the rTMS treatment to treat the severe OCD and behavioral symptoms which affected her functionality and her interactions with family and others to a great extent. These severe symptoms put herself at risk (such as cutting her hair by herself) in an OCD manner for no reason, with temper tantrums. The outweighed risk from rTMS was lower and was managed by a further reduction of the intensity of the treatment.

Following the first seizure, the patient was diagnosed with unspecified encephalopathy by a neurologist. Regarding her medication, she reduced the clonazepam dose and started taking sodium valproate. A new seizure episode occurred 10 days after her last TMS session with this new reduced intensity and the abovementioned changes in her medication. This second seizure episode happened at her school area. The situation of the patient resolved without medication or hospitalization. The characteristics of the second seizure as well as its duration were the same as the first one. However, the second seizure episode seemed to be unrelated to the rTMS therapy because it occurred 10 days after her last rTMS session and after a change of her medication.

\section{Discussion}

In this case report, we present a patient who experienced a seizure while she was receiving HF-rTMS to the DMPFC using a double-cone TMS coil for treating her OCD symptoms. This patient had been treated already in the past using rTMS for her depressive and anxiety symptoms using a standard figure- 8 TMS coil targeting the more superficial DLPFC. This depression rTMS treatment was well tolerated, and no serious adverse events were reported.

Syncope is a condition often misdiagnosed as epileptic seizure. Syncope is "a transient loss of consciousness caused by transient global cerebral hypoperfusion characterized by rapid onset, short duration, and spontaneous complete recovery" [13]. The most possible reasons for this misdiagnosis are the following: syncope affects a large percentage of the population (around 40\%), syncope may mimic the clinical presentation of epileptic seizures, and finally, epileptic seizures and syncope may coexist in a patient [14]. Differentiating

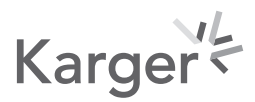


epileptic seizure from syncope can be difficult; however, there are several clinical tips to find the correct diagnosis. In the present case report, we discriminate epileptic seizure from syncope using the following clinical information. First of all, the duration of both episodes lasted around 2-3 min. Regarding seizures, generalized tonic-clonic seizure lasts $30 \mathrm{~s}-5 \mathrm{~min}$, and secondarily, generalized tonic-clonic seizure lasts $62 \mathrm{~s}$ (16 s-108s), whereas syncope has fewer duration, around 15 s (3 s-30 s) [15]. In epileptic seizures, falls are characterized as fast and tonic, in contrast to syncope where falls are slow and flacid [15]. The patient experienced tongue-biting, a symptom that is presented rarely in syncope, while is a common symptom in epileptic seizure $[11,13]$. Another point that differs between epileptic seizure and syncope is the eye deviation. Lateral eye deviation is more common in epileptic seizures, whereas fixed or upward eye deviation in syncope. As for heart rate, syncope is associated with bradycardia [11], contrastingly the patient in the present case report experienced tachycardia following both episodes. Finally, her postictal phases lasted around $10 \mathrm{~min}$, which is a typical period of time between the end of a seizure and the return to baseline situation. Her postictal phases characterized with symptoms like confusion and headache. Confusion is the most common symptom of the postictal phase of epileptic seizure in contrast to syncope where the most frequent symptoms are brief haziness, fatigue, diaphoresis, and nausea [13]. During her OCD treatment, however, the first seizure episode occurred during the 7 th session using the recently FDA-approved protocol for OCD targeting the DMPFC using a double-cone coil with high-frequency rTMS [9]. In a study by Carmi et al. [9], 89 patients were treated with this protocol, and no seizure cases were reported. To our knowledge, until today, there have been no reported seizures using this FDA-approved protocol for OCD. As regards the stimulation frequency, Chou et al. [12] suggested that around half of the reported seizure cases until February 2020 occurred during HF-rTMS. Contrarily, Lerner et al. [10] noted that HF-rTMS was no more likely to cause seizures than LF-rTMS and single/paired-pulse TMS when applied in patients without risk factors. Taking into account those previous studies, it thus remains unclear whether HF-rTMS is the main or only driving factor for an increased risk for seizure induction compared with other TMS protocol parameters. However, according to the safety and recommendations for TMS use in healthy subjects and patient populations [11], "any type of person with any pattern of stimulation might have a seizure."

During the last decades, different types of TMS coils have been developed with different geometries and stimulation properties. The main types of TMS coils are figure-of- 8 coil, circular coil, double-cone coil, and $\mathrm{H}$ coil. The figure-of- 8 coil is the most commonly used coil in TMS treatment. It is characterized by 2 adjacent wings, and it was designed to provide more focal stimulation of cortical regions, below the central part of the coil. The double-cone coil is characterized by 2 large adjacent circular wings at an angle of $95^{\circ}$ [11]. Double-cone coils have the ability to stimulate deeper brain areas and produce a higher magnetic field with higher depth of penetration in contrast to standard TMS coils such as figure-of-8 coils [16]. According to a recent survey, double-cone coils are associated with only 1 seizure case [10]. In 2021, a review on safety of use of rTMS in clinical practice and research has been published reporting no serious adverse events using the double-cone coil [11]. The risk for seizure induction using the double-cone coil is $0.12 / 1,000$, while using the figure-of- 8 coil is $0.08 / 1,000$ [10]. Double-cone coils are less frequently used in rTMS practice compared with the figure-of- 8 coil. This may be a reason why TMS-induced seizures are less frequent while using the double-cone coil. Our patient, in contrast, did experience a seizure during the first sessions of this novel OCD TMS protocol using a double-cone coil over DMPFC. Interestingly, this patient was experienced with rTMS, undergoing 30 sessions of theta burst rTMS to the DLPFC using a figure-8 coil without any adverse effects. This suggests that the rTMS-induced seizure in her case is related to the change in target region or coil type. Nevertheless, there are several other predisposing factors for the occurrence of seizure in psychiatric patients

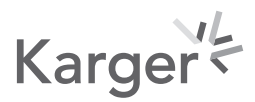


such as pharmacotherapy that may affect seizure thresholds, substance consumption such as alcohol, caffeine, and some instable behavioral patterns such as agitation and sleep deprivation [11]. However, as in all naturalistic settings and most patients, this conclusion may not be the only possible interpretation. It is noteworthy that this patient also experienced a second seizure episode, a few days after the last rTMS session was applied. Therefore, the second seizure episode appears to be unrelated to the rTMS therapy and possibly related to this change of her medication. Also, during and following this rTMS treatment, an unspecific brain anomaly was detected in her MRI scan, potentially putting her a posteriori in a certain risk group of patients for rTMS. This of course does not explain the well-tolerated depression TMS treatment she received prior to the OCD TMS protocol.

\section{Conclusion}

This seizure induction may be the first reported seizure using the recently FDA-approved protocol for OCD targeting the DMPFC with a double-cone TMS coil. This seizure was most likely triggered by rTMS. At this point, we want to nonetheless report this case in order to increase awareness that with new protocols and new coil types, also the evaluation of tolerability needs to be updated and closely monitored. Despite its acceptability and increasing popularity, TMS should still be administered by clinicians or TMS technicians who are aware of such cases and prepared to follow all the appropriate recommendations and seizure management protocols during the rTMS treatment. Future reviews are needed to clarify further the mechanisms that may trigger seizure episodes during rTMS treatment (patient characteristics, TMS parameters, target region, and TMS coil) in order to reduce the likelihood of rTMS-induced seizures.

\section{Statement of Ethics}

The research was conducted ethically in accordance with the World Medical Association Declaration of Helsinki. As this was a single retrospective case report, we did not seek a bioethical approval; however, a relevant informed consent was obtained. The relevant consent for publication could be provided upon request. The subject in this study gave written informed consent for publication of her case including publication of images.

\section{Conflict of Interest Statement}

The authors have no conflicts of interest to declare.

\section{Funding Sources}

The authors received no specific funding for this work.

\section{Author Contributions}

All authors agree with the contents of the manuscript and were fully involved in the study and preparation of the manuscript. All authors have read the final version of the manuscript and have approved the submission. George Mikellides and Panayiota Michael 
drafted the manuscript. Teresa Schuhmann and Alexander T. Sack helped to draft the manuscript and supervised the project.

\section{Data Availability Statement}

All data generated or analyzed during this study are included in this article. Further enquiries can be directed to the corresponding author.

\section{References}

1 Berlim MT, van den Eynde F, Tovar-Perdomo S, Daskalakis ZJ. Response, remission and drop-out rates following high-frequency repetitive transcranial magnetic stimulation (rTMS) for treating major depression: a systematic review and meta-analysis of randomized, double-blind and sham-controlled trials. Psychol Med. 2014 Jan;44(2):225-39.

2 Haghighi M, Shayganfard M, Jahangard L, Ahmadpanah M, Bajoghli H, Pirdehghan A, et al. Repetitive Transcranial Magnetic Stimulation (rTMS) improves symptoms and reduces clinical illness in patients suffering from OCD: results from a single-blind, randomized clinical trial with sham cross-over condition. J Psychiatr Res. 2015 Sep;68:238-44.

3 Klomjai W, Katz R, Lackmy-Vallée A. Basic principles of transcranial magnetic stimulation (TMS) and repetitive TMS (rTMS). Ann Phys Rehabil Med. 2015 Sep;58(4):208-13.

4 Berlim MT, Neufeld NH, Van den Eynde F. Repetitive transcranial magnetic stimulation (rTMS) for obsessivecompulsive disorder (OCD): an exploratory meta-analysis of randomized and sham-controlled trials. J Psychiatr Res. 2013 Aug;47(8):999-1006.

5 Hawken ER, Dilkov D, Kaludiev E, Simek S, Zhang F, Milev R. Transcranial magnetic stimulation of the supplementary motor area in the treatment of obsessive-compulsive disorder: a Multi-Site Study. Int J Mol Sci. 2016 Mar 22;17(3):420.

6 Ruffini C, Locatelli M, Lucca A, Benedetti F, Insacco C, Smeraldi E. Augmentation effect of repetitive transcranial magnetic stimulation over the orbitofrontal cortex in drug-resistant obsessive-compulsive disorder patients: a controlled investigation. Prim Care Companion J Clin Psychiatry. 2009;11(5):226-30.

7 Elbeh KAM, Elserogy YMB, Khalifa HE, Ahmed MA, Hafez MH, Khedr EM. Repetitive transcranial magnetic stimulation in the treatment of obsessive-compulsive disorders: double blind randomized clinical trial. Psychiatry Res. 2016 Apr 30;238:264-9.

8 U.S. Food and Drug Administration. [Internet] FDA permits marketing of transcranial magnetic stimulation for treatment of obsessive compulsive disorder. [cited 2018 August 17]. Available from: https://www.fda.gov/ news-events/press-announcements/fda-permits-marketing-transcranial-magnetic-stimulation-treatmentobsessive-compulsive-disorder.

9 Carmi L, Tendler A, Bystritsky A, Hollander E, Blumberger DM, Daskalakis J, et al. Efficacy and safety of deep transcranial magnetic stimulation for obsessive-compulsive disorder: a Prospective Multicenter Randomized Double-Blind Placebo-Controlled Trial. Am J Psychiatry. 2019 Nov 1;176(11):931-8.

10 Lerner AJ, Wassermann EM, Tamir DI. Seizures from transcranial magnetic stimulation 2012-2016: results of a survey of active laboratories and clinics. Clin Neurophysiol. 2019 Aug;130(8):1409-16.

11 Rossi S, Antal A, Bestmann S, Bikson M, Brewer C, Brockmöller J, et al. Safety and recommendations for TMS use in healthy subjects and patient populations, with updates on training, ethical and regulatory issues: expert guidelines. Clin Neurophysiol. 2021 Jan;132(1):269-306.

12 Chou YH, Ton That V, Chen AY, Sundman M, Huang YZ. TMS-induced seizure cases stratified by population, stimulation protocol, and stimulation site: a systematic literature search. Clin Neurophysiol. 2020 May; 131(5):1019-20.

13 Sheldon R. How to differentiate syncope from seizure. Cardiol Clin. 2015 Aug;33(3):377-85. PMID: 26115824.

14 Ungar A, Ceccofiglio A, Pescini F, Mussi C, Tava G, Rafanelli M, et al. Syncope and epilepsy coexist in "possible" and "drug-resistant" epilepsy (overlap between Epilepsy and Syncope Study - OESYS). BMC Neurol. 2017 Feb 28;17(1):45.

15 Kowacs PA, Silva Júnior EB, Santos HL, Rocha SB, Simão C, Meneses MS, et al. Syncope or epileptic fits? Some examples of diagnostic confounding factors. Arq Neuropsiquiatr. 2005 Sep;63(3A):597-600.

16 Schecklmann M, Schmaußer M, Klinger F, Kreuzer PM, Krenkel L, Langguth B. Resting motor threshold and magnetic field output of the figure-of-8 and the double-cone coil. Sci Rep. 2020 Feb 3;10(1):1644. 